\section{Un réseau qui donne du relief à l'hypophyse}

Xavier Bonnefont, Alain Lacampagne,

Nathalie Courtois-Coutry, Iain C.A.F. Robinson, Patrice Mollard

$>$ L'hypophyse antérieure constitue la glande maîtresse de notre système endocrine. À ce titre, elle synthétise et sécrète dans la circulation générale plusieurs hormones essentielles à un grand nombre de fonctions physiologiques aussi importantes que la croissance et le métabolisme (GH et TSH), la reproduction et la lactation (LH, FSH, PRL) ou encore le stress (ACTH). Comme pour la plupart des systèmes endocrines, l'efficacité d'action de ces hormones dépend largement de leur profil de sécrétion au cours du temps. La prise de conscience de cette donnée temporelle représente une avancée majeure en endocrinologie médicale. Ainsi, l'injection d'une dose unique et massive d'hormone de croissance $(\mathrm{GH})$ est nettement moins efficace pour le traitement de certains déficits de croissance que des injections multiples de doses faibles, mimant le rythme de sécrétion endogène de GH $[1,2]$. Quels mécanismes sont mis en œuvre par notre organisme pour donner à l'hypophyse son caractère pulsatile, essentiel à son efficacité ?

Depuis la période riche en événements scientifiques des années 1950-1970 [3], nous savons que l'hypothalamus contrôle les sécrétions hypophysaires par des neuro-hormones libérées de façon pulsatile dans le système porte hypothalamo-hypophysaire. Par exemple, le rythme de GH résulte probablement des sécrétions anti-parallèles de neurohormones activatrice (GHRH) et inhibitrice (SRIF) [4]. Cependant, si l'hypothalamus impose un certain tempo aux sécrétions hypophysaires, un phénomène d'intégration dans l'hypophyse joue vraisemblablement un grand rôle dans l'amplitude des pics hormonaux qui ne peut être expliquée par la simple somme des activités unitaires. Dans une étude récente [5], nous avons montré que les cellules somatotropes (sécrétant la GH) de l'hypophyse peuvent communiquer via un réseau anatomique câblant toute la glande.

\section{La vision en 3D révèle l'inattendu}

Des travaux princeps des histologistes ayant identifié les différents types endocrines et non endocrines de l'hypophyse $[6,7]$, nous avons hérité une image plane de la glande, dans laquelle les cellules endocrines sont dispersées au sein du parenchyme, sans apparente organisation. Grâce à la microscopie à excitation 2-photons, qui permet une exploration en profondeur des tissus biologiques [8], nous avons étudié la distribution en 3D des cellules somatotropes dans l'hypophyse de souris exprimant une protéine fluorescente (GFP) sous contrôle du promoteur du gène de la GH [9]. Ces cellules GH-GFP, apparemment réparties au hasard dans le plan, apparaissent en fait liées les unes aux autres dans la profondeur de la glande. Elles forment ainsi un vaste «squelette endocrine» dont l'existence demeurait insoupçonnée jusqu'alors [5] (Figure 1).

\section{Robustesse et plasticité}

Bien loin d'être le fruit du hasard, l'architecture de ce réseau GH-GFP est
X. Bonnefont, N. Courtois-Coutry, P. Mollard : Institut de Génomique Fonctionnelle, Département Endocrinologie, CNRS UMR 5203, Inserm U661, Universités Montpellier 1 et 2, 141, rue de la Cardonille,

34094Montpellier Cedex 05, France.

A Lacampagne: Inserm U637,

CHU Arnaud de Villeneuve,

34295Montpellier Cedex 05, France.

I.C.A.F. Robinson:

Division of Molecular Neuroendocrinology,

NIMR, The Ridgeway, Mill Hill,

London NW7 1AA, Royaume-Uni.

Xavier.Bonnefont@igf.cnrs.fr

Patrice.Mollard@igf.cnrs.fr

reproductible d'un animal à l'autre. Par exemple, chez les souris mâles, les cellules GH-GFP sont réparties sur de longues lignes cellulaires dans la partie médiane de l'hypophyse alors qu'elles se regroupent en amas denses dans les zones latérales. Ce caractère stéréotypé suggère la robustesse de l'architecture du réseau GH-GFP, par ailleurs démontrée par la présence de cadhérines protéines impliquées dans la formation des jonctions adhérentes - entre les cellules GH-GFP.

Malgré sa structure robuste, cet édifice cellulaire n'est pas figé au cours de la vie de l'animal et fait même preuve d'une réelle plasticité en fonction de la demande en GH. En effet, les amas GHGFP des zones latérales n'apparaissent chez les souris mâles qu'au moment de la maturation sexuelle, lorsque la sécrétion de GH et la croissance corporelle sont à leur maximum. Après cette phase aiguë, le système reprend une organisation similaire à celle observée lors des premières étapes post-natales. Cette plasticité architecturale est absente dans la glande d'animaux castrés avant la puberté. Ces 
animaux présentent par ailleurs un déficit de croissance bien que la densité moyenne de cellules GH-GFP soit la même que chez les animaux témoins. Ainsi, plus que le nombre ou la densité de cellules $\mathrm{GH}$ dans la glande, c'est bien leur distribution dans l'espace qui est importante pour une fonctionnalité optimale.

\section{Une connectivité}

fonctionnelle à grande échelle

Comment l'arrangement en réseau 3D des cellules GH peut-il conduire à une sécrétion plus efficace de GH? Pour tenter de répondre à cette question, nous avons mesuré la concentration en calcium cytosolique dans des cellules individuelles au sein de tranches d'hypophyse. En fonction de l'organisation locale entre les cellules GH-GFP, deux types de réponses au GHRH ont pu être distinguées [5]. D’une part, dans les zones latérales où les cellules GH sont fortement condensées, des vagues calciques lentes apparaissent en même temps dans la majorité (sinon toutes) des cellules GH. Le mécanisme res- ponsable d'un tel phénomène demeure inconnu mais révèle la capacité des cellules GH-GFP à harmoniser leur activité à grande échelle. D'autre part, les cellules $\mathrm{GH}$ alignées dans la zone médiane semblent répondre au GHRH de façon beaucoup moins coordonnée, à l'exception de quelques paires de cellules qui présentent un couplage total de leur activité, probablement grâce à l'ouverte de jonctions communicantes [10]. Ces observations mettent en évidence un lien étroit entre distribution spatiale des cellules et codage temporel de l'activité cellulaire et intercellulaire. La synchronisation de l'activité observée dans les zones latérales pourrait vraisemblablement permettre d'optimiser le fonctionnement des cellules à l'échelle de la glande et ainsi augmenter l'efficacité d'action de la GH sécrétée.

Le réseau GH-GFP fait écho au réseau de communication non-endocrine formé par les cellules folliculo-stellaires dans I'hypophyse antérieure [11]. Ces données démontrent la structuration fonc-

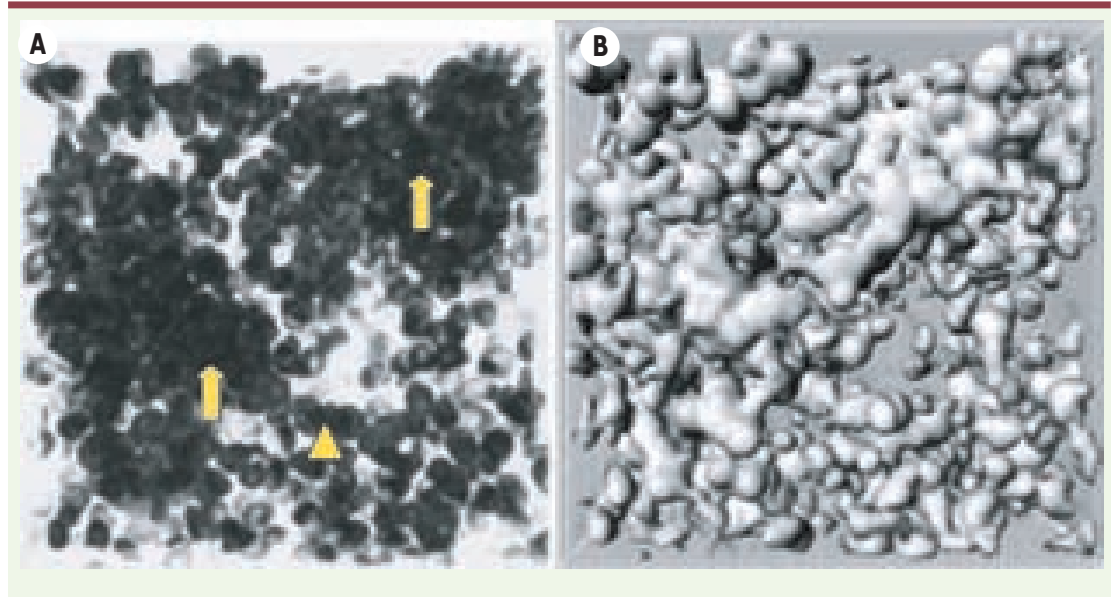

Figure 1. Réseau de cellules GH-GFP dans l'hypophyse. A. L'imagerie en profondeur des cellules GH-GFP révèle leur distribution en amas denses (仓) ou en lignes de cellules $(\triangle$ ). $B$. Ces deux structures forment un vaste continuum visible grâce à la projection 3D des images de microscopie à excitation 2-photons.

tionnelle de la glande et suggèrent que d'autres types cellulaires, dans l'hypophyse ou même d'autres tissus endocrines, pourraient suivre une distribution spatiale particulière en fonction de la demande en hormone ou de l'état physiopathologique. Grâce aux progrès en imagerie médicale nous pourrons peutêtre bientôt suivre l'évolution de ces réseaux endocrines chez les patients et ainsi établir des diagnostics précoces d'hypofonctionnement endocrine ou de tumorigenèse. $\diamond$

A 3D cell network which brings out relief in the pituitary gland

\section{RÉFÉRENCES}

1. Clark RG, Chambers G, Lewin J, Robinson IC. Automated repetitive microsampling of blood: growth hormone profiles in conscious male rats. J Endocrinol $1986 ; 111: 27-35$.

2. Thorner M0, Reschke J, Chitwood J, et al. Acceleration of growth in two children treated with human growth hormone-releasing factor. $N$ Engl J Med 1985; 312: 4-9.

3. Rostène W. Claude Fortier : the great history of neuroendocrinology. Med Sci (Paris) 2005 ; 21 : 551-5.

4. Tannenbaum GS. Genesis of episodic growth hormone secretion. J Pediatr Endocrinol 1993; 6 : 273-82.

5. Bonnefont X, Lacampagne A, Sanchez-Hormigo A, et al. Revealing the large-scale network organization of growth hormone-secreting cells. Proc Natl Acad Sci USA 2005 ; 102 : 16880-5.

6. Herlant M. Study of the pituitary body with the periodic acid-Schiff reaction. Nature 1949; $164: 703$

7. Vila-Porcile $\varepsilon$. The network of the folliculo-stellate cells and the follicles of the adenohypophysis in the rat (pars distalis). Z Zellforsch Mikrosk Anat 1972; $129: 328-69$

8. Denk W, Svoboda K. Photon upmanship : why multiphoton imaging is more than a gimmick. Neuron $1997 ; 18: 351-7$.

9. Magoulas C, McGuinness L, Balthasar N, et al. A secreted fluorescent reporter targeted to pituitary growth hormone cells in transgenic mice. Endocrinology $2000 ; 141$ : 4681-9.

10. Guérineau NC, Bonnefont X, Stoeckel L, Mollard P. Synchronized spontaneous $\mathrm{Ca}^{2+}$ transients in acute anterior pituitary slices. J Biol Chem 1998; 273 : 10389-95.

11. Fauquier T, Guérineau NC, McKinney RA, et al. Folliculostellate cell network : a route for longdistance communication in the anterior pituitary. Proc Natl Acad Sci USA 2001 ; 98 : 8891-6. 